\title{
A game theoretic framework for rate allocation and charging of Available Bit Rate (ABR) connections in ATM networks
}

\section{H. Yaiche}

Département de Génie Electrique et Génie Informatique, Ecole Polytechnique de Montréal

C.P. 6079, succ. A, Montreal, Quebec, Canada H3C $3 A 7$

Telephone: +(514) 340-4123, Fax: +(514) 340-4562,

Email: yaiche@comm.polymtl.ca

\section{R.R. Mazumdar}

Department of Mathematics, University of Essex

Wivenhoe Park, Colchester, United Kingdom CO4 $3 S Q$

Telephone: +(1206) 873-032, Fax: +(1206) 873-043,

Email:mazum@essex.ac.uk

\section{Rosenberg}

Nortel plc, Harlow, and Department of Electrical and Electronic Engineering, Imperial College, U.K.

London Road, Harlow, United Kingdom CM17 9NA

Telephone: +(1279) 403 889, Fax: +(1279) 402400 ,

Email: caro@nortel.co.uk

\begin{abstract}
In this paper we present an abstract, rigorous game-theoretic framework for rate-based control of Available Bit Rate (ABR) services in ATM networks. The framework is based on the idea of the Nash arbitration scheme in cooperative game theory which not only provides the rate settings of users which are Pareto optimal from the network point of view of but are also consistent with the fairness axioms of game theory. We first consider the centralized problem and then show that this procedure can be de-centralized such that users can perform the optimization locally such that the overall rates are network optimal. We then consider the problem of charging of ABR connections considering users' valuations for the best-effort service. We propose a flat-rate charging policy such that the total network revenue is maximum. we show that the above arbitration set-up can be used to characterize a rate alloca-
\end{abstract}




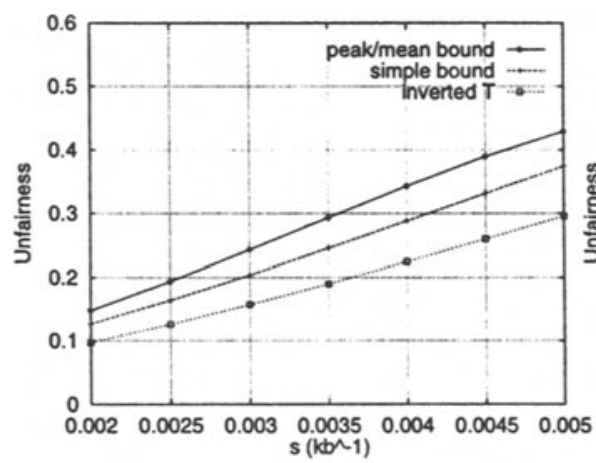

(a) $B=0.25 \times 10^{6}$ bytes

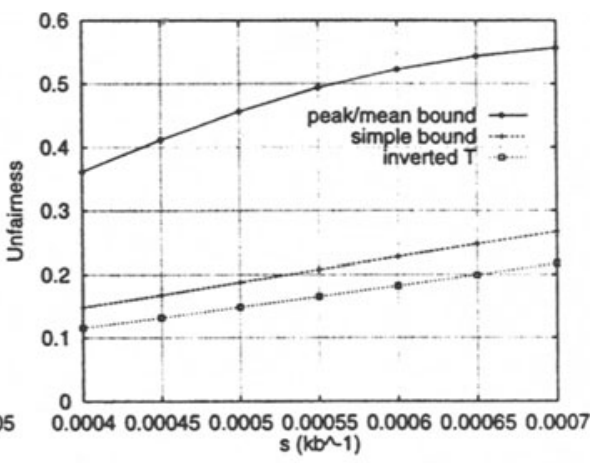

(b) $B=1 \times 10^{6}$ bytes

Figure 3 Unfairness for $C=34 \mathrm{Mbps}$ and two buffer sizes.

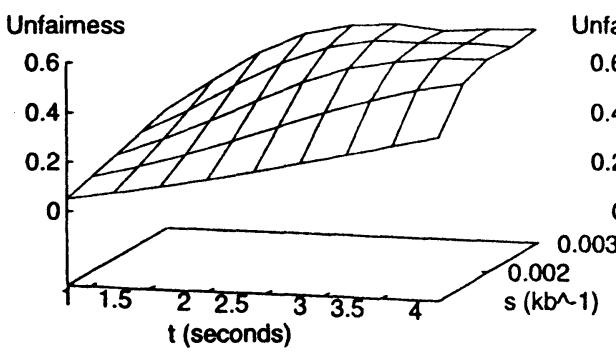

(a) peak/mean bound

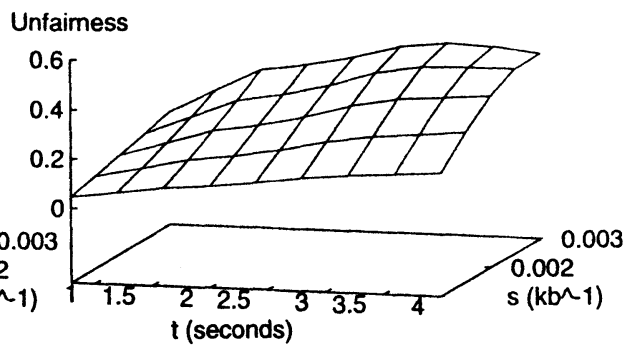

(b) simple bound

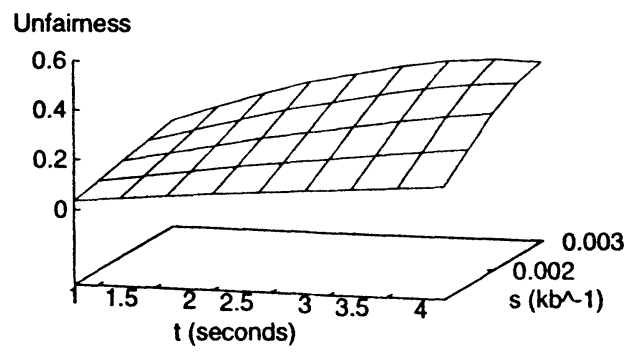

(c) inverted T

Figure 4 Unfairness for $C=34 \mathrm{Mbps}, B=0.25 \times 10^{6}$ bytes. 
tion policy which takes into consideration users' bandwidth requirements and users' valuations in a fair way.

\section{Keywords}

ABR capability, explicit rate control, game theory, fairness, willingness-to-pay

\section{INTRODUCTION}

The ITU-T [10] has introduced a new ATM Transfer category (ATC) called the Available Bit Rate (ABR) ATC to support data applications which cannot be efficiently supported by existing bandwidth guaranteed ATC's. The ABR ATC has been targetted to support highly bursty applications which have no way of predicting their traffic requirements in advance but which have welldefined cell loss requirements and can tolerate time-varying and unpredictable transfer delays. Another characteristic of these applications is that they are able to modify their data transfer rates according to network conditions. This encapsulates the notion of elastic traffic services by which the source rates are adjusted according to the network conditions in order that the network can carry or pack as many revenue generating connections subject to some minimal guarantees.

These applications are expected to ride "on top of" bandwidth guaranteed connections and utilize any excess bandwidth. Since the available bandwidth will change depending on the amount of "background" bandwidth guaranteed services being carried the incoming elastic sources will have to continually change their rates based on some notification by the network on the available bandwidth. Thus the notion of rate control of sources arises.

The study of rate control in the context of ABR services has been receiving much attention recently since the success of ATM networks will be crucially dependent on the ability of such networks to carry internet data type applications. Since there will be many sources which will be competing for the use of the available bandwidth there are several issues which arise and must be dealt with to have an efficient means of allocating available network resources. These are: 1) efficient bandwidth allocation to the different sources taking into account their different performance requirements 2) the crucial notion of fairness 3 ) the notion of decentralized or local rate control in order to facilitate the control to be implemented on a per-connection or local procedure 4) and finally the charging and its relationship with bandwidth allocation.

The problem of rate control in the context of ABR services has been studied in terms of incremental schemes whereby the rate of sources is adjusted by an additive increase factor in case of notification by the network that more bandwidth is available and is decremented by a multiplicative factor on notification by the network that it is becoming congested. A recent paper by Hernandez-Valencia et al [9] presents a survey of the current state of the art. 
The issue of fairness is addressed in that the rate settings are max-min fair. Other approaches to the problem can be found in the recent special issue [7].

In a recent important paper, Kelly [11] addresses the bandwidth allocation or the rate settings of the sources by considering explicit utility functions for the performance. In particular the paper addresses two important issues : those of optimal rate settings from a network point of view which corresponds to a constrained optimization of the sum of the utility functions as well as the problem of local user optimization whose solution corresponds to the network optimum. By considering logarithmic utility functions Kelly then goes on to show that such a scheme results in rate settings which are proportionally fair (i.e. if a source needs twice a given amount of bandwidth compared to another source it will receive twice the amount of bandwidth which is allocated to achieve the netwprk optimum). In a follow-up paper, Kelly et al [12] show that a real-time algorithm can be developed which converges to the required rate settings which can be used by individual sources and provide a detailed probabalistic sample-path analysis. The basic idea is that the equilibrium state of the differential system is precisely the required rates and they show that the basic property of the algorithm is that it acts as a Lyapunov function for the system.

This paper is motivated by the issues raised in the paper of Kelly [11]. In particular, by drawing upon ideas from cooperative game theory [8] it is shown that the idea proposed by Kelly is in fact a Nash arbitration scheme [14] which has the property that it is a Pareto optimal and hence qualifies as a network optimal scheme while satisfying certain axioms of fairness. Once this observation is made the abstract framework then allows us to address the ABR problem with non-zero minimum cell rate (MCR) requirements while also accounting for peak cell rate (PCR) constraints of sources. This allows us to address the rate allocation problem from the point of view of performance characteristics rather than abstract utility functions. In particular we show that a local procedure can be devised such that the solution of the local optimization corresponds to the arbitrated solution in the Nash sense. We then consider the problem of charging ABR connections considering users' valuations. In this context, we propose a network rate allocation policy which takes into account users' bandwidth requirements and users' valuations in a fair way.

The idea of the Nash arbitration scheme in the context of telecommunication networks is not new. This was first presented in the context of packetswitched (data) networks by Mazumdar et al [13]. The properties of Pareto optimality as well as the development of local optimization procedures which lead to Pareto optimal solutions (the local procedures being greedy schemes) was studied in a series of papers by Douligeris and Mazumdar [4], and [5] in the context of data networks. This paper is thus an extension of those ideas as well as a new approach in the context of ABR (or elastic) services in ATM networks. 
The outline of this paper is as follows: in Section 1 we present the salient facts about the Nash arbitration scheme which provides the framework of the solution. Section 2 considers the optimal and fair rate allocation problem for ABR connections in which we discuss both the centralized (network optimality) as well as the connection based (local algorithm) contexts. In section 3 we introduce the charging framework. Section 4 concludes the paper. Throughout we omit details of proofs since they readily follow from standard results in optimization theory.

\section{NASH ARBITRATION SCHEMES}

In this section we present the salient concepts and results from cooperative game theory and the Nash arbitration schemes which are used in the sequel. For details we refer the reader to the book by Fudenberg and Tirole [8] and the paper of Nash [14].

The basic setting of the problem is as follows: there are $N$ users (connections) which compete for the use of a fixed resource (bandwidth). Each user $i(i \in\{1 . . N\})$ has a performance function $f_{i}$ and an initial performance $u_{i}^{0}$. Each performance function is defined on a subset of $\mathcal{R}^{N}$ termed $X$ which is the set of game strategies of the $N$ users. In a context of network resource allocation $X$ could represent the space of allocated rate vectors. The initial performance of each user represents a minimum performance that a user wants to achieve and will not enter the game if it is not possible to realize it. Therefore, we will assume throughout our theoretical framework that each user involved in the game can achieve its initial performance. In other words, there exists at least a vector in $X$ such that the value at that point of the agregate performance function, $f,\left(\left(f_{1}, \ldots, f_{N}\right)\right)$ is superior or equal to the initial performance vector, $u^{0}$.

In our framework we adopt the following mathematical assumptions regarding a user's performance function and the space of strategies. Indeed, $X$ is assumed to be nonempty, convex, and closed set. The functions $f_{i}$ are assumed to be real-valued, upper-bounded, and concave functions. Finally a word about notation: given two vectors $u, v \in \mathcal{R}^{N}$ we say $u \geq v$ if $u_{i} \geq v_{i} \forall i \in\{1,2, \ldots, N\}$.

Let $U \subset \mathcal{R}^{N}$ be a nonempty convex closed and upperbounded set. Let $u^{0} \in \mathcal{R}^{N}$ such that $U_{0}=\left\{u \in U / u \geq u^{0}\right\} \neq \emptyset$. Let $G_{k}=\left\{\left(U, u^{0}\right) / U \subset \mathcal{R}^{N}\right.$ is a nonempty convex closed and upperbounded set and $u^{0} \in \mathcal{R}^{N}$ such that $\left.U_{0} \neq \emptyset\right\} . G_{k}$ is a set of pairs. Each pair is characterized by a set and an initial point.

We first define the notion of Pareto optimality in the context of multiplecriteria objectives which occurs in the typical game setting with multiple players.

Definition 2.1 The point $u \in U$ is said to be Pareto-optimal if for each $u \prime \in U, u \prime \geq u$ it implies that $u \prime=u$.

The interpretation of a Pareto optimum is that it is impossible to find 
another point which leads to strictly superior performance for all the players simultaneously. In general in a game with $\mathrm{N}$ players (or equivalently for a set of N-objectives) the Pareto optimal points form a $\mathrm{N}$ dimensional hypersurface which implies that there are an infinite number of points which are Pareto optimal. From the definition of Pareto optimality it is clear that a network optimal operating point must be a Pareto optimal point. The question that arises is which of (the infinitely many) Pareto optimal points must the network be operated?

One way in which we can define suitable Pareto optimal points for operation is by introducing further criteria. From the perspective of resource sharing one of the natural criteria is the notion of fairness. This in general is a loose term and there are many notions of fairness. One of the commonly used notions is that of max-min fairness which corresponds to a saddle-point for the game and is most commonly used in the context of ABR control [9]. However a much more satisfactory approach is to use the fairness axioms from game theory as the fairness criteria [14].

We now define the Nash arbitration scheme which encapsulates the above requirements.

Definition 2.2 $A$ mapping $S: G_{k} \rightarrow \mathcal{R}^{N}$ is said to be a Nash arbitration scheme if:

1. $S\left(U, u^{0}\right) \in U_{0}$.

2. $S\left(U, u^{0}\right)$ is Pareto-optimal.

3. S satisfies linearity axiom; If $\phi: \mathcal{R}^{N} \rightarrow \mathcal{R}^{N}, \phi(u)=u$ ' with $u \prime_{j}=a_{j} u_{j}+b_{j}$, $a_{j}>0, j=1, \ldots, N$ then $S\left(\phi(U), \phi\left(u^{0}\right)\right)=\phi\left(S\left(U, u^{0}\right)\right)$.

4. $S$ satisfies irrelevant alternatives axiom; If $U_{\prime} \subset U,\left(U \prime, u^{0}\right) \in G_{k}$, and $S\left(U, u^{0}\right) \in U$ ' then $S\left(U, u^{0}\right)=S\left(U \prime, u^{0}\right)$.

5. $S$ satisfies symmetry axiom; If $U$ is symmetrical with rspect to a subset $J \subseteq\{1, \ldots, N\}$ of indices (i.e. $u \in U$ and $i, j \in J, i<j$ imply $\left.\left(u_{1}, \ldots, \bar{u}_{i-1}, u_{j}, u_{i+1}, \ldots, u_{j-1}, u_{i}, u_{j+1}, \ldots, u_{N}\right) \in U\right)$, and if $u_{i}^{0}=u_{j}^{0} i, j \in J$ then $S\left(U, u^{0}\right)_{i}=S\left(U, u^{0}\right)_{j} i, j \in J$.

Having defined the Nash arbitration scheme we define the optimal point as follows:

Definition 2.3 Let $U=\left\{u \in \mathcal{R}^{N} / \exists x \in X\right.$ such that $\left.f(x) \geq u\right\}$. Let $u^{*}$ be given by $S\left(U, u^{0}\right)$. Then $u^{*}$ is the (Nash) arbitration point and $f^{-1}\left(u^{*}\right)$ is called the set of the (Nash) arbitrated solution.

Remark 2.1 The items 3, 4 and 5 above are the so-called axioms of fairness. The linearity property of the solution implies that the arbitration scheme is scale invariant i.e. the arbitrated solution is unchanged if the performance objectives are scaled. The irrelevant alternatives axiom states that the arbitration point is not affected by enlarging the domain while the symmetry property states that the arbitration point does not depend on the specific labels i.e. users with the same initial points and objectives will realize the same performance. 
The following result due to Stefanescu [15] provides for a characterization of the Nash arbitration point and will form the basis for the results in the sequel.

Theorem 2.1 Let $U=\left\{u \in \mathcal{R}^{N} / \exists x \in X\right.$ such that $\left.f(x) \geq u\right\}$. Denote by $X(u)=\{x \in X / f(x) \geq u\} . X_{0}=X\left(u^{0}\right)$, the subset of strategies that enable the users to achieve at least their initial performances.

Then there exists a unique arbitration scheme and a unique arbitration point $u^{*}$. Moreover the set of the arbitrated solution $\left(f^{-1}\left(u^{*}\right)\right)$ is determined as follows:

Let $J$ be the set of users able to achieve strictly better than their initial performance i.e., $J$ is defined as $\left\{j \in\{1 . . N\} / \exists x \in X_{0}, f_{j}(x)>u_{j}^{0}\right\}$. Each strategy vector $x$ in the arbitrated solution set verifies $f_{J}(x)>u_{J}^{0}$ and solves the following maximization problem $\left(P_{J}\right)$ :

$$
\left(P_{J}\right) \quad M a x \prod_{j \in J}\left(f_{j}(x)-u_{j}^{0}\right) \quad x \in X_{0}
$$

Hence, $u^{*}$ satisfies that $u_{j}^{*}>u_{j}^{0}$ for $j \in J$ and $u_{j}^{*}=u_{j}^{0}$, otherwise.

Remark 2.2 Note that for each $j \in \bar{J}, \forall x \in X_{0} f_{j}(x)=u_{j}^{0}$. Also, it can be readily shown that if each function $f_{j}(j \in J)$ is injective on $X_{0}$ then the arbitrated solution set is a singleton and therefore there exists a unique Nash arbitrated solution strategy vector.

We now state an equivalent optimization problem which will also result in a Nash arbitration scheme and which we will consider in context of rate allocation for ABR connections.

Theorem 2.2 In addition to the assumptions in 2.1, let $\left\{f_{j}\right\} ; j \in J$ be injective on $X_{0}$.

Consider the two maximization problems $\left(P_{J}\right)$ and $\left(P_{J}\right)$ :

$$
\begin{array}{cccc}
\left(P_{J}\right) & \operatorname{Max} & \prod_{j \in J}\left(f_{j}(x)-u_{j}^{0}\right) & x \in X_{0} \\
\left(P \prime_{J}\right) & \operatorname{Max} & \sum_{j \in J} \ln \left(f_{j}(x)-u_{j}^{0}\right) & x \in X_{0}
\end{array}
$$

Then:

(i) $\left(P_{J}\right)$ has a unique solution; The arbitrated solution set is a singleton.

(ii) $\left(\mathrm{P}_{J}\right)$ is a convex program and has a unique solution.

(iii) $\left(P_{J}\right)$ and $\left(P_{J}\right)$ are equivalent. Hence, the unique solution of $\left(P_{J}\right)$ is the arbitrated solution.

Remark 2.3 In [11] Kelly considers the centralized optimization criterion as the weighted sum of the logarithmic utility functions and hence the corresponding optimal solution is a Nash arbitration solution in light of the above result. 


\section{OPTIMAL AND FAIR RATE ALLOCATION FOR ABR CONNECTIONS}

We propose a scheme that results in an optimal and fair share of the available bandwidth between ABR connections competing for network resources. The scheme is based on the game theory framework presented above. Each user (connection) has a performance function to be maximized. Each function is defined on the space of the allocated rate vectors. We characterize an optimal and fair operating point as a solution of the optimization of a global network's objective function.

It is natural to adopt a game theory approach to model and address the issue of network resource allocation. In the context of flow control in packetswitched networks many schemes were based on the use of game theory and gave a characterization for some candidate points. Some of them considered Nash equilibrium points [2] [5] and others considered Pareto-optimal points [6]. In [13], the Nash arbitration point was proposed as a suitable solution for the design of an optimal and fair flow control.

As in [13] we consider the Nash arbitration point as a desired point for the operation of the network. This is due to the Pareto optimality and fairness property associated with Nash arbitration schemes.

The definition of a Nash arbitration point is highly dependent on the consideration of an initial performance point (termed $u^{0}$ in the previous section). It represents a minimum performance that a user wants to achieve and the user will not enter the game if it is not possible. In the context of Available Bit Rate service (ABR), for each connection (user) the initial performance can be viewed as a performance achieved by the Minimum Cell Rate (MCR) guaranteed by the network.

We now introduce a utility function for each user (connection), $U$, that depends on the allocated rate vector. It represents user's satisfaction from a particular performance level achieved through an allocated rate vector. The determination of the Nash arbitrated solution give us a natural candidate for a utility function. Indeed, we assume that a user $i$ with a performance function $f_{i}$ and a minimum desired performance $u_{i}^{0}$ has a utility function $U_{i}$ defined as follows:

$U_{i}(x)=\ln \left(f_{i}(x)-u_{i}^{0}\right) \quad x \in X_{0}$.

The network's global objective function to be maximized is the overall satisfaction (or the sum of the utilities) of the users able to achieve strictly better than their initial performance $\left(u_{i}^{0}\right.$ for user $\left.i\right)$. The choice is motivated by the fact that the maximization of the considered global function leads to the desired fair and optimal operating point; the Nash arbitration point.

The results presented in this section are meant to be applied for the implementation of a rate-based control mechanism for Available Bit Rate (ABR) transfer capability. We propose a criterion for optimal and fair rate allocation based on the Nash arbitration scheme which takes into account both zero and 
non-zero-minimum-cell-rate ABR connections. This formulation copes with the limitations of the max-min fairness schemes, [1], which apply unambiguously only to ABR connections with minimum cell rate equal to zero.

Firstly, we present a centralized model in which network resources are the available link capacities and each $\mathrm{ABR}$ connection aims at maximizing its allocated rate beyond its minimum cell rate. The centralized model identifies a global optimization problem from which the Nash arbitrated solution (allocated rate vector) emerges as the unique solution. Secondly, we propose a decomposed or decentralized model in which each connection and the network provider are separate entities and have their own optimization criteria. We show that by appropriate choice of network parameters the Nash arbitrated solution of the centralized model is an optimal allocated rate vector for each connection and the network provider. The decomposed model is a necessary step to develop a distributed algorithm of a rate-based control mechanism implementing the Nash arbitrated solution criterion.

\subsection{Centralized model}

We consider a static model for the centralized (network) problem in which $N$ connections are established. Each connection corresponds to an ABR connection with a Peak Cell Rate (PCR), a Minimum Cell Rate (MCR) guaranteed by the network, and an assigned path. Connections compete for available bandwidth resources within the network. These resources are network link available capacities and they are assumed to be fixed (non-time-varying). With respect to the abstract framework already presented the allocated rate vector space, $X$, is determined by network capacity constraints and connections' peak cell rates. It is defined as follows:

$X=\left\{x \in \mathcal{R}^{N} / x \geq 0 \quad x \leq P C R\right.$ and $\left.A x \leq C\right\}$.

where $C$ is the vector of link capacities , PCR is the vector of peak cell rates of the connections, and $A=\left(a_{l p}\right)_{l, p}$ is a $L \times N$ incidence matrix i.e. $a_{l p}$ is equal to 1 if the link $l$ belongs to the path $p$ and 0 otherwise.

In the context of Available Bit Rate it is natural to assume that each connection aims to maximize its throughput (and so its allocated rate) beyond its minimum cell rate. Therefore, with respect to the abstract framework the performance function, $f_{i}$, for a user $i$ is simply defined as $x_{i}$. Moreover, $M C R_{i}$ represents the initial and the minimum performance desired by user $i$.

We assume that the initial performance vector (the $M C R_{i}$ 's) is achievable which means that on each network link the spare capacity is superior to the sum of the $M C R_{i}$ 's of the connections crossing this link. The set of the achieving rate vectors, $X_{0}$, is characterized as follows:

$X_{0}=\left\{x \in \mathcal{R}^{N} / x_{i} \geq M C R_{i} x_{i} \leq P C R_{i}\right.$ and $\left.A x \leq C\right\}$.

For simplicity and without loss of generality, we assume that on each link the spare capacity is strictly superior to the sum of the $M C R_{i}$ 's of the connections 
crossing this link. If this assumption is not valid then our model and results are still valid for the subset of connections to which we can allocate more than the corresponding minimum cell rate. One can show that this assumption ensures that $X_{0}$ has a nonempty interior.

With respect to the abstract framework, the Nash arbitrated solution of the centralized model is an optimal and fair rate allocation of network available capacities to the $N$ considered ABR connections. It is the unique solution of the following global optimization convex problem $(S)$ :

$\left\{\begin{array}{l}\operatorname{Max}_{\{x\}} \prod_{i=1}^{N}\left(x_{i}-M C R_{i}\right) \\ x \in X_{0}\end{array}\right.$

\subsection{Decomposed or decentralized model}

In the previous section we formulated and solved the centralized network optimal rate allocation problem. In general this will involve centralized coordination amongst the ABR connections. Thus a challenging issue is that can such a problem be decentralized by which connections perform only a local optimization such that the locally optimized rates allocated are optimal in a global or network sense?

We propose a decomposed model in which each user (connection) and the network provider is a seperate entity. In this model, each connection can vary its connection rate, freely. The rate for the connection is bounded from below by the MCR and from above by the PCR. Each connection has a utility function measuring the satisfaction achieved through a particular rate. Moreover each user's global satisfaction experiences a decrease as a result of accessing and using network resources. Indeed, we introduce $N$ positive network parameters, $\alpha_{i}$ 's, which represent the decrease per unit rate of the global satisfaction of the $N$ users given that they share the resources. $\alpha_{i}$ is also the benefit per rate unit realized by the network provider as a result of user $i$ accessing its resources. Therefore, the objective of each user is to maximize its net satisfaction which is, for a particular rate, the difference between the utility and the cost of accessing the network. Hence, each user $i$ solves the following strictly convex problem $\left(U_{i}\right)$ :

$\left\{\begin{array}{l}\operatorname{Max}_{\left\{x_{i}\right\}} U_{i}(x)-\alpha_{i} x_{i} \\ x_{i}>M C R_{i} \\ x_{i} \leq P C R_{i}\end{array}\right.$

The network provider aims to determine the optimal rate allocation to users that maximizes its total benefit. Hence, it has to solve the following convex problem $(N)$ :

$\left\{\begin{array}{l}\operatorname{Max}_{\{x\}} \sum_{i=1}^{N} \alpha_{i} x_{i} \\ x \in X_{0}\end{array}\right.$

The following proposition shows that by appropriate choice of network para- 
meters, the $\alpha_{i}$ 's, the Nash arbitrated solution of the centralized model maximizes each user's net satisfaction and the network total benefit.

Proposition 3.1 Let $x$ be the unique Nash arbitrated solution of the centralized problem $(S)$. Then, there exist positive real numbers $\alpha_{i}(i \in\{1 . . N\})$ such that $x$ is the unique solution of the problems $\left(U_{i}\right)$ and $x$ is a solution of the problem $(N)$.

It is interesting to notice that if we view a user's utility function in terms of monetary value, then we obtain an economic and a pricing framework of rate allocation. In this framework, a utility function will have the interpretation of a willingness-to-pay for a particular rate. The $\alpha_{i}$ 's will stand for the prices of a rate unit charged to users. Each user will aim to maximize its net benefit and the network (provider) will aim to maximize its total revenue.

In the following section we show how the game theoretic framework can be used to derive a charging mechanism for ABR and an allocation policy taking into account users' valuations in a fair way.

\section{A CHARGING SCHEME FOR ABR USERS}

We propose to address the issue of rate allocation together with the charging issue in the context of ABR considering users' bandwidth requirements and users' valuations for the best-effort service. Each user informs the network of its valuation either at subscription time or at connection set-up. Indeed, a user chooses a price it is willing to pay for any excess allocated bandwidth beyond the guaranteed minimum cell rate. The price may be chosen from a given set of values or from a set of pre-determined tariffs published by the network provider. Different users with different peak and minimum cell rates can choose from different sets of price values. The choice of a user reflects the value it attaches to any excess bandwidth allocated by the network. There is an agreement between users and the network by which the network assures that its allocation policy will allocate bandwidth optimally and fairly taking into account their bandwidth constraints as well as their willingness-to-pay.

We propose a network allocation policy and a two-components charging policy. First, a user is charged for its guaranteed minimum cell rate according to a tariff function which may depend on both minimum and peak cell rate parameters. The second component is a flat-rate charge that a user pays for any excess allocated bandwidth. The network chooses to charge each user its willingness-to-pay as in this case the total revenue is the maximum possible that the network can get from any bandwidth allocation.

The network adopts an allocation policy which takes into account users' willingness-to-pay ( $\beta_{i}$ for user $i$ ) for excess bandwidth in a fair way. This particular policy satisfies a desirable property in that the more a user is willing to pay for the excess bandwidth the more allocated bandwidth it gets. The rate settings is characterized as the Nash arbitrated solution of a cooperative game with the user performance objectives defined as the power function of the rate allocated above the MCR for each user with exponent $\beta_{i}$ (assumed to 
be between 0 and 1). The reason behind the consideration of such allocation policy is that it incorporates nice fairness properties with regard to users' valuations. Considering a model of $N$ users (connections) similar to the one described in section 3.1, the rate settings is determined as follows:

- If $\beta_{i}=0$ then $x_{i}=M C R_{i}$ otherwise

- If $\sum_{l=1}^{L} \mu_{l} a_{l i}>0$ then $x_{i}=M C R_{i}+M I N\left(P C R_{i}-M C R_{i}, \frac{\beta_{i}}{\sum_{l=1}^{L} \mu_{l} a_{l i}}\right)$.

- If $\sum_{l=1}^{L} \mu_{l} a_{l i}=0$ then $x_{i}=P C R_{i}$.

Where the $\mu_{l}$ 's $(l \in\{1 . . L\})$ stand for the link shadow prices associated with the game-underlying optimization problem. If we view these prices as the allocation costs of a bandwidth unit to users then it can be readily seen that the allocation policy has the following nice characteristics:

- If a user $i$ pays nothing for the share of bandwidth beyond the minimum cell rate then its allocated rate is the minimum cell rate.

- If a user $i$ pays for the share of bandwidth beyond the minimum cell rate then its allocated rate is greater than the minimum cell rate.

- If the nework's resources along a user's path are free then its allocated rate is the peak cell rate.

- If the nework's resources along a user's path are not free and but the user's valuation exceeds the network path cost per unit of bandwidth by more than a factor of $(P C R-M C R)$ then the user is allocated its peak rate.

- If the nework's resources along a user's path are not free and the user's price is less than the path cost per bandwidth unit then the user is allocated a cell rate between the minimum and peak rate proportional to the valuation of the user. As a result, if two users share the same resources and one of them is willing to pay the double of the other, then the user receives double the share of bandwidth beyond minimum cell rate.

- If two users share the same resources, have the same maximum excess bandwidth (difference between peak and minimum cell rates), and are willing to pay the same price then they get the same share of excess bandwidth.

\section{CONCLUDING REMARKS}

In this paper we have presented a game theoretic framework for the allocation of optimal rates to ABR connections which share common bandwidth. This framework allows us to go further in showing how we can come up with a charging scheme and an allocation policy which presents nice fair properties. A point to note is that the framework is a static one. The main aim has been to point out how the problem of rate allocation can be addressed within the framework of the Nash arbitration scheme. An important issue to be addressed in the future is the development of a distributed algorithm which implements the Nash arbitrated allocation policy using the ABR Explicit Rate (ER) mechanism. 


\section{Acknowledgement}

The authors would like to acknowledge useful insights provided by Prof. F. P. Kelly. This research has been supported in part by contracts from the Centre National d'Etudes des Telecommunications (CNET), France through the Consultations Thématiques (CTI) programme and by the CANCAN project of ACTS by the European Economic Community.

\section{REFERENCES}

[1] D. Bertsekas and R. Gallager: Data networks. Prentice-Hall, 1987.

[2] K. Bharathkumar and J.M. Jaffe: $A$ new approach to performance oriented flow control. IEEE Trans. Comm., vol. COM-29, April 1981, pp. 427-435.

[3] F. Bonomi and K. W. Fendick: The rate-based flow control framework for available bit rate ATM services, IEEE Network, March/April, 1995.

[4] C. Douligeris and R. Mazumdar: On Pareto-optimal flow control in an integrated environment. Proc. 25th Allerton Conf., Univ. Illinois, Urbana, October 1986.

[5] C. Douligeris and R. Mazumdar: User optimal flow Control in an integrated environment. Proc. Indo-U.S. Workshop on Syst. Signals, Bangalore, India, January 1988.

[6] P. Dubey: Inefficiency of Nash equilibria. Math. Oper. Res., vol. 11, no. 1 , pp. 1-8, 1986.

[7] European Transactions on Telecommunications, Focus on Elastic Services over ATM networks, R. Mazumdar and B. Doshi eds., ETT Vol. 8, N0. $1,1997$.

[8] D. Fudenberg and J. Tirole: Game theory, MIT Press, Cambridge, Ma., 1995.

[9] E. J. Hernandez-Valencia, L. Benmohammed, S. Chong and R. Nagarajan: Rate-control algorithms for ATM ABR service, in [10], pp. 7-20.

[10] ITU-T Recommendation I.371: Traffic control and congestion control in B-ISDN, Geneva, June 1996.

[11] F. Kelly: Charging and Rate Control for Elastic Traffic. in [10], pp. 33-37

[12] F. Kelly, A. Maulloo and D. Tan: Rate control in communication networks: shadow prices, proportional fairness and stability, pre-print, Statistical Lab., Cambridge, 1997

[13] R. Mazumdar, L. Mason, and C. Douligeris: Fairness in network optimal flow control: optimality of product forms. IEEE Transactions on communications, vol. 39 , no. 5 , 1991, pp. 775-782.

[14] J. Nash: The bargaining problem, Econometrica, Vol. 18, 1950, pp. 155162 .

[15] A. Stefanescu and M.W. Stefanescu: The arbitrated solution for multiobjective convex programming. Rev. Roum. Math. Pure. Appli., vol. 29, pp. 593-598, 1984. 